\title{
Xp11.2 translocation renal cell carcinoma with egg-shell calcification mimicking a benign renal tumour: A case report
}

\author{
WENJIE LIANG and SHUNLIANG XU \\ Department of Radiology, The First Affiliated Hospital, College of Medicine, Zhejiang University, \\ Hangzhou, Zhejiang 310003, P.R. China
}

Received November 4, 2014; Accepted August 20, 2015

DOI: $10.3892 / \mathrm{ol} .2015 .3718$

\begin{abstract}
The present study reports the case of a 20 -year-old female who was identified to have a left renal angiomyolipoma (AML) with hemorrhage. Following temporary conservative observation, the patient received continuous ultrasonic follow-up. Due to the rapid growth of the lesion, further examinations were performed. Computed tomography (CT) plain scans revealed a partly high-density mass with marginal egg-shell calcification. Enhanced CT revealed a solid tumor with a rich blood supply. Since no fats were detected, the possibility of a typical AML was excluded, but the diagnoses of epithelioid AML or renal cancer were considered. Finally, the left kidney was partially excised laparoscopically. The intraoperative frozen section indicated a diagnosis of renal cell carcinoma (RCC). The left kidney was subsequently radically excised. . Routine histopathological and immunohistochemical tests confirmed that the lesion was an RCC with an Xp11.2 translocation. The present study introduces the pitfalls in the diagnosis of Xp11.2 translocation RCC, which is a rare RCC subtype accompanied with uncommon imaging manifestations. The study suggests that when a rapidly-growing AML is detected by ultrasound, renal cancer with marginal calcification should be considered. Moreover, although egg-shell calcification mostly occurs in benign renal lesions, further examinations, such as enhanced CT, are recommended for identifying the nature of the masses and excluding the possibility of malignant tumors.
\end{abstract}

\section{Introduction}

Renal cell carcinoma (RCC) is the most common malignant renal tumor originating from tubular epithelioid cells. In

Correspondence to: Dr Wenjie Liang, Department of Radiology, The First Affiliated Hospital, College of Medicine, Zhejiang University, 79 Qingchun Road, Hangzhou, Zhejiang 310003, P.R. China

E-mail: baduen.c@163.com

Key words: renal cancer, renal angiomyolipoma, computed tomography, ultrasound, egg-shell calcification the United States, RCC accounts for 3\% of all tumors (1). The tumors are most likely to occur among males who are $\sim 60$ years old $(2,3)$. The most common clinical manifestations include hematuria, bellyache and a lateral abdominal mass, but only $\sim 10 \%$ of cases present with this typical triad (4). The incidence rate of RCC is stably increasing, and one major reason for this is an increased detection rate due to gradually enhanced diagnostic imaging capabilities $(5,6)$. Ultrasound and enhanced computed tomography (CT) are commonly used in the examination and evaluation of renal masses, owing to their advantages in the detection and staging of tumors, and in post-operative follow-up. Xp11.2 translocation RCC is an uncommon type of $\operatorname{RCC}(7,8)$. This type of RCC has a more invasive clinical progression in adults with a worse prognosis compared with other types of RCC. Therefore it is important to become familiar with the imaging characteristics of this particular type of $\operatorname{RCC}(7,8)$. The present study reports the case of an Xp11.2 translocation RCC with egg-shell calcification that was misdiagnosed as renal angiomyolipoma (AML).

\section{Case report}

Three months ago, a left renal mass was identified in a 20 -year-old female by ultrasound examination. The patient presented with a $>3$-month history of lower abdominal pain, lower back pain and drinking-induced abdominal distension, and $\mathrm{a} \geq 1$-month history of frequent urination and hematuria. Three continuous ultrasound examinations in the subsequent 2 months revealed the rapid growth of the lesion.

Enhanced CT indicated the possibility of epithelioid AML. The possibility of renal cancer (Bosniak IV type) could not be excluded. On June 28th, 2013, the patient was admitted to The First Affiliated Hospital of Zhejiang University (Hangzhou, China) for further evaluation and treatment. There was no history of central nervous system disease, kidney disease, hypertension or other chronic diseases, and the patient had no relevant bad habits. Upon physical examination, there was no swelling in the lower limbs, no pain on percussion in the kidneys, no pain on palpation of the bilateral ureteral paths. Bladder insufficiency and dull bladder percussion were noted. Renal routine examinations revealed a glomerular filtration rate (MDRD equation) of $126.0 \mathrm{ml} / \mathrm{min}$ (normal range, $80.0-125.0 \mathrm{ml} / \mathrm{min}$ ), $56 \mu \mathrm{mol} / 1$ creatinine (normal range, 45.0 $84.0 \mu \mathrm{mol} / \mathrm{l}$ ), $3.5 \mathrm{mmol} / \mathrm{l}$ urea (normal range, $2.9-8.2 \mathrm{mmol} / \mathrm{l}$ ) 


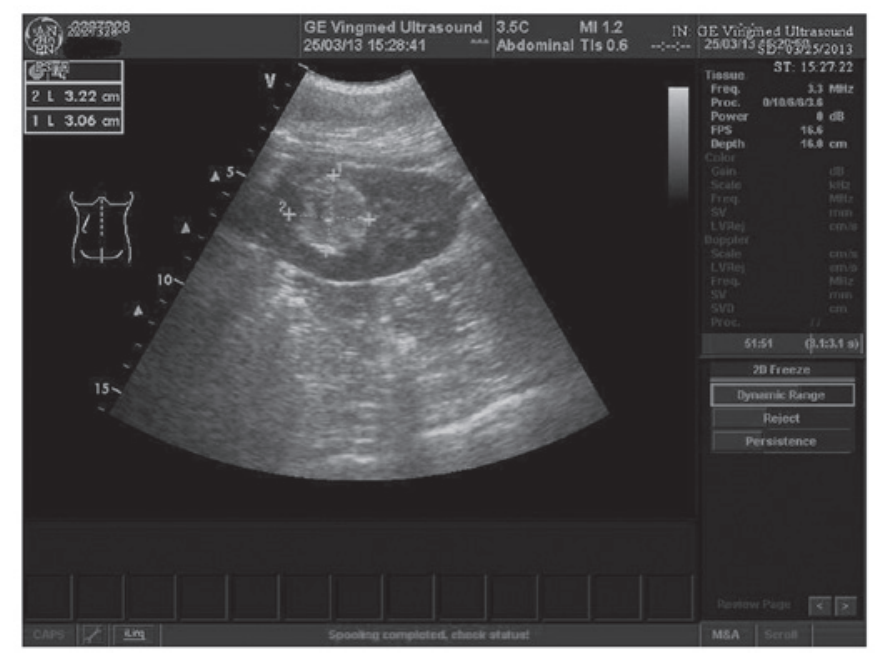

Figure 1. Abdominal ultrasound examination showing high echoes from the upper pole of the left kidney to the medulla. The echoes were alternately weak then strong, followed by attenuation.

and $269 \mu \mathrm{mol} / \mathrm{l}$ uric acid (normal range, 155.0-357.0 $\mu \mathrm{mol} / \mathrm{l}$ ). Urine routine examinations revealed occult blood $+(1.0) \mathrm{mg} / \mathrm{l}$ (normal, $0 \mathrm{mg} / \mathrm{l}$ ) and glucose $\pm(1.7) \mathrm{mmol} / \mathrm{l}$ (normal, $0 \mathrm{mmol} / \mathrm{l}$ ). Other laboratory test indices were all normal.

The patient received three ultrasound examinations and one enhanced CT kidney examination. The first ultrasound examination confirmed a strong-echo mass with sizes of $3.2 \times 3.1 \mathrm{~cm}^{2}$ in the upper pole of the left kidney on March 25th, 2013 (Fig. 1). Due to the strong-weak alternating echoes inside and the partial echoless areas observed locally, AML accompanied with hemorrhage was first considered. The repeat ultrasound revealed continuous mass with sizes of $3.4 \times 3.0 \mathrm{~cm} 2$ in the left kidney on April 22th, 2013. The third ultrasound revealed the left renal mass with sizes of $3.6 \times 3.0 \mathrm{~cm} 2$ on May 23th, 2013, indicating that the tumor was growing rapidly. Next, enhanced CT was performed to further clarify the nature of the mass. CT plain scans revealed that the upper pole of the left kidney was occupied, while the margins of the lesion were affected with linear egg-shell-like calcifications, but with relatively higher density in the upper lesion and lower density in the lower region (Fig. 2A). The enhanced CT scan revealed that the upper region of the lesion was significantly enhanced at the early stage, but that later the enhancement was slightly reduced. The lower region was not enhanced (Fig. 2B and C).

The enhanced CT clarified the solid components with a rich blood supply. Since no fat tissues were detected, the possibility of classic AML was reduced. Despite the probability of epithelioid AML, the possibility of renal cancer was increased. Thus, active surgical treatment was implemented.

At first, the patient underwent close conservative observation, as the lesion was rather small and ultrasonically manifested as characteristic AML. Since the mass grew rapidly, enhanced CT was implemented. The possibility of renal cancer could not be excluded. According to the surgical plan, a partial nephrectomy was first performed laparoscopically. RCC rather than AML was confirmed by intraoperative frozen section with the finding that the tumor was composed of obviously heteromorphic transparent cells. A radical nephrectomy was subsequently performed, which showed that
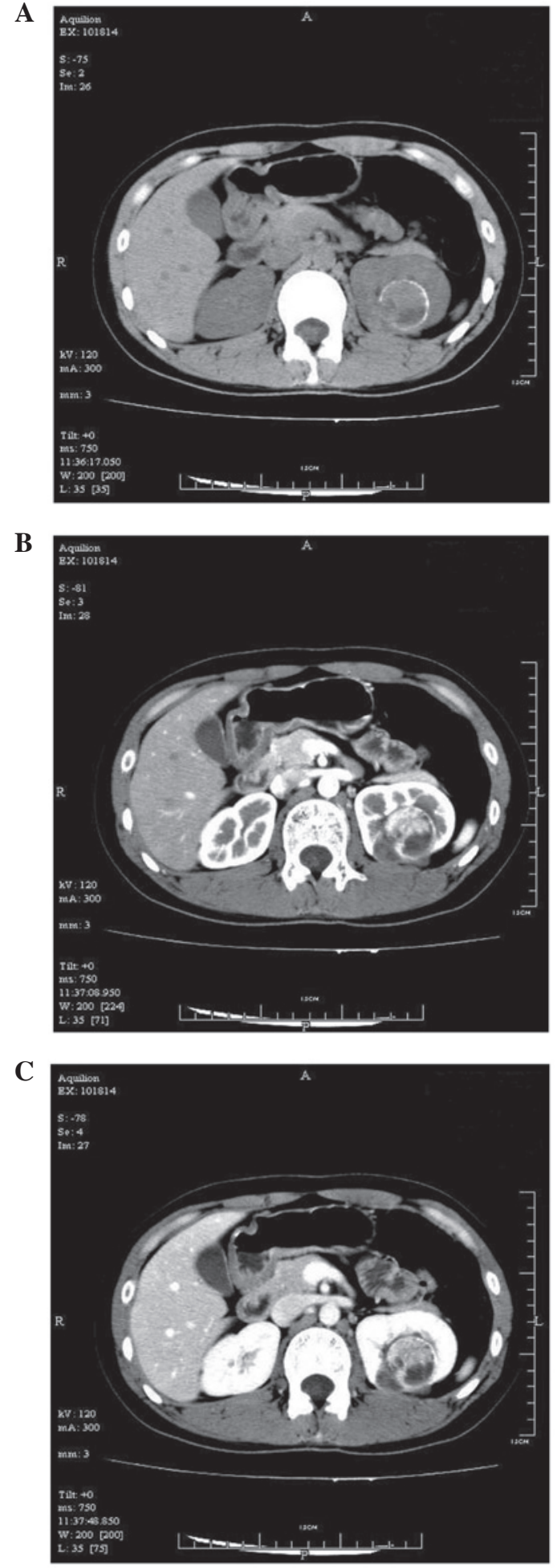

Figure 2. Kidney CT. (A) CT plain scan showing masses with marginal thin calcification in the left renal mass; the solid region is slightly high density (50.4 HU) and partially cystic; (B) enhanced CT indicating that the solid region at arterial phase is markedly enhanced $(126.7 \mathrm{HU})$; (C) the enhancement at venous phase is slightly reduced $(107.3 \mathrm{HU})$ and the cystic part is not enhanced (B and C). CT, computed tomography.

tumor was nest-shaped with papillary arrangement, invasive growth and obvious heteromorphic cells; the cytoplasm was transparent and blood sinuses were enriched in the stroma. Immunohistochemistry demonstrated the expression level of CK(pan) (partly+), Vimentin (+), CK7(focus+), CD10 (+), 
E-cadherin (-), CD117 (-), TFE3 (+), HMB45 (-), Melan-A (-). Routine histopathological and immunohistochemical tests confirmed that the lesion was an RCC with Xp11.2 translocation. The patient recovered well post-operatively. During the 12-month follow-up, no recurrence or metastases were found.

\section{Discussion}

Renal carcinoma associated with Xp11.2 translocations/TFE3 gene fusions is a rare RCC subtype, according to the 2004 World Health Organization tumor classification (7). The clinical manifestations and laboratory tests for this tumor are not specific, and in the majority of cases, indicate incidental asymptomatic renal masses (8). Compared with regular RCCs, Xp11.2 translocation RCC usually occurs among children and young adults, particularly females $(8,9)$. Compared with other RCCs, its clinical process is more invasive with a poorer prognosis (10). Furthermore, the imaging manifestations of Xp11.2 translocation RCC are diverse, including hemorrhage, necrosis, cystic degeneration and calcification $(8,11-15)$. Therefore, the pre-operative diagnosis of this entity is occasionally difficult, as in the present case.

Due to its safety and accessibility, ultrasound examination is used as a general means for screening renal masses. Ultrasound will differentiate the components of a lesion (e.g. calcification, necrosis, hemorrhage or fat) by displaying the differences between transmitted waves. Ultrasound is fairly accurate for the diagnosis of renal AML, and the typical manifestations are markedly higher echoes accompanied with rear acoustic shadowing, as it is mixed with several tissue components (e.g. fats) and contains rich vascular nets $(16,17)$. In the present case, however, the lesion was hyperechoic, and was combined with the sound shadow behind the marginal calcification, all of which simulated the ultrasonic manifestations of a typical AML. Moreover, the necrotic cystic degeneration was reasonably explained as hemorrhage. Due to the limitations of the ultrasound machine, the blood supply of the mass was not observed. The first ultrasound examination was completed by a urinary ultrasound expert with $>30$ years of experience, and thus, the misdiagnosis of this case deserves serious attention.

CT scans with high-density resolution aid in the differentiation between tissue components, such as calcification, hemorrhage, soft tissues, fats, cystic degeneration or necrosis. In the present case, the different components of the mass were displayed clearly by enhanced CT, including the marginal calcification, the solid components with rich blood supply and the unenhanced necrotic region. Since no fat was detected, the diagnosis was targeted around epithelioid AML and renal cancer. Based on previous findings, calcification inside a mass indicates a high probability of malignant lesions, while egg-shell calcification usually occurs in benign lesions (simple cysts) $(18,19)$. In the present case, the imaging manifestations on the CT plain scan mimicked a cyst with calcification and chronic hematoma, but the enhanced scan showed marked enhancement in the tissues within the tumor, thus revealing the essence of the tumor. Therefore, a simple CT plain scan is inadequate for the evaluation of renal lesions accompanied with egg-shell calcification, and further evaluation of components inside the lesion is essential. AML is occasionally difficult to differentiate from RCC; usually AML contains a varying amount of fats, which is a key differential point (20-22). In the present case, the egg-shell calcification partly misguided the diagnosis, but two possible diagnoses were provided, which ensured that a reasonable surgical therapy was applied for the misdiagnosed patient.

In general, this case of Xp11.2 translocation RCC with egg-shell calcification was ultrasonically manifested as AML. However, the enhanced CT scan revealed egg-shell calcification and a rich-blood-supply mass without fat. This study suggests that when an ultrasound indicates AML, the differential diagnosis should include the possibility of renal cancer accompanied with egg-shell calcification. When a CT plain scan shows a high-density cyst with egg-shell calcification, the differential diagnosis should also include the possibility of renal cancer. Enhanced CT will aid in the complete evaluation of the nature of a renal mass with egg-shell calcification.

\section{References}

1. Jemal A, Siegel R, Ward E, Murray T, Xu J and Thun MJ: Cancer statistics. CA Cancer J Clin 57: 43-66, 2007.

2. Luciani LG, Cestari R and Tallarigo C: Incidental renal cell carcinoma-age and stage characterization and clinical implications: Study of 1092 patients (1982-1997). Urology 56: 58-62, 2000.

3. Aron M, Nguyen MM, Stein RJ and Gill IS: Impact of gender in renal cell carcinoma: An analysis of the SEER database. Eur Urol 54: 133-140, 2008

4. Zweizig SL: Cancer of the kidney. Clin Obstet Gynecol 45: 884-891, 2002.

5. Hollingsworth JM, Miller DC, Daignault S and Hollenbeck BK: Rising incidence of small renal masses: A need to reassess treatment effect. J Natl Cancer Inst 98: 1331-1334, 2006.

6. Chow WH, Devesa SS, Warren JL and Fraumeni JF Jr: Rising incidence of renal cell cancer in the United States. JAMA 281: 1628-1631, 1999.

7. Lopez-Beltran A, Scarpelli M, Montironi R and Kirkali Z: 2004 WHO classification of the renal tumors of the adults. Eur Urol 49: 798-805, 2006

8. Wang W, Ding J, Li Y, Wang C, Zhou L, Zhu H and Peng W: Magnetic resonance imaging and computed tomography characteristics of renal cell carcinoma associatedwith Xp11.2 translocation/TFE3 gene fusion. PLoS One 9: e99990, 2014.

9. Winarti NW, Argani P, De Marzo AM, Hicks J and Mulyadi K: Pediatric renal cell carcinoma associated with Xp11.2 translocation/TFE3 gene fusion. Int J Surg Pathol 6: 66-72, 2008.

10. Camparo P, Vasiliu V, Molinie V, Couturier J, Dykema KJ, Petillo D, Furge KA, Comperat EM, Lae M, Bouvier R, et al: Renal translocation carcinomas: Clinicopathologic, immunohistochemical and gene expression profiling analysis of 31 cases with a review of the literature. Am J Surg Pathol 32: 656-670, 2008.

11. Dang TT,Ziv E, Weinstein S, Meng MV, Wang Z and Coakley FV: Computed tomography and magnetic resonance imaging of adult renal cell carcinoma associated with Xp11.2 translocation. J Comput Assist Tomogr 36: 669-674, 2012.

12. Koo HJ, Choi HJ, Kim MH and Cho KS: Radiologic-pathologic correlation of renal cell carcinoma associated with Xp11.2 translocation. Acta Radiol 54: 827-834, 2013.

13. Liu K, Xie P, Peng W and Zhou Z: Renal carcinomas associated with Xp11.2 translocations/TFE3 gene fusions: Findings on MRI and computed tomography imaging. J Magn Reson Imaging 40: 440-447, 2014.

14. He J, Huan Y, Qiao Q, Zhang J and Zhang JS: Renal carcinomas associated with Xp11.2 translocations: Are CT findings suggestive of the diagnosis? Clin Radiol 169: 45-51, 2014.

15. Kmetec A and Jeruc J: Xp 11.2 translocation renal carcinoma in young adults; recently classified distinct subtype. Radiol Oncol 48: 197-202, 2014.

16. Lienert AR and Nicol D: Renal angiomyolipoma. BJU Int 110: 25-27, 2012. 
17. Halpenny D, Snow A, McNeill G and Torreggiani WC: The radiological diagnosis and treatment of renal angiomyolipoma-current status. Clin Radiol 165: 99-108, 2010.

18. Daniel WW Jr, Hartman GW, Witten DM, Farrow GM and Kelalis PP: Calcified renal masses. A review of ten years experience at the Mayo Clinic. Radiology 103: 503-508, 1972.

19. Dyer RB, Chen MY and Zagoria RJ: Abnormal calcifications in the urinary tract. Radiographics 18: 1405-1424, 1998.

20. Kim JY, Kim JK, Kim N and Cho KS: CT histogram analysis: Differentiation of angiomyolipoma without visible fat from renal cell carcinoma at CT imaging. Radiology 246: 472-479, 2008.
21. Sasiwimonphan K, Takahashi N, Leibovich BC, Carter RE, Atwell TD and Kawashima A: Small $(<4 \mathrm{~cm})$ renal mass: Differentiation of angiomyolipoma without visible fat from renal cell carcinoma utilizing MR imaging. Radiology 263: 160-168, 2012.

22. Chung MS, Choi HJ, Kim MH and Cho KS: Comparison of T2-weighted MRI with and without fat suppression for differentiating renal angiomyolipomas without visible fat from other renal tumors. AJR Am J Roentgenol 202: 765-771, 2014. 